\title{
Analyzing Carrier to Noise Interference Ratio on Performance Enhancement for Dynamic Channel Allocation Scheme
}

\author{
Suyash Kumar' ${ }^{1}$, P V Suresh ${ }^{2}$ \\ SOCIS IGNOU, New Delhi, India.
}

\begin{abstract}
In this paper, using dynamic channel allocation scheme, we have examined the carrier to noise ratio concerned with the system and by conducting channel search based on finding an associated low carrier to noise ratio, we show that our system provides a low blocking probability. We have examined various performance evaluation parameters show that our scheme improves the system performance under various parameter settings.
\end{abstract}

Keywords: Dynamic channel algorithm, blocking Probability, Co-channel interference, etc.

\section{INTRODUCTION}

The demand for wireless communication has consistently exceeded the capacity of available technologies. A problem essentially unique to wireless network is the extremely hostile and random nature of radio channel and uncertainty in the demand of channels by users. Since the users may request service from any physical location while traveling over a wide range of velocities, the network switches are forced to hand over calls imperceptibly between base stations throughout the system. The radio spectrum available for wireless network is limited, and hence is constrained to operate in a fixed bandwidth to support increasing number of users and high data rate demands. As the wireless network grows, the necessary addition of base station increases the switching burden on the network switches. One of the prime issues in such network design is how to use the channels effectively for communication purpose. Another issue is interference due to massive number of users and other parameters. Unlike wired communications which benefits from isolation provided by cables, wireless users within close proximity can cause considerable interference to one another. Hence to overcome such factors, the concept of cellular wireless mobile network was introduced where each cell is allocated a portion of the total frequency spectrum. For efficient utilization of the radio spectrum, frequency reuse schemes that are consistent with the objectives of increasing capacity and minimizing interferences are required. Frequency reuse is a technique of reusing frequencies and channels within a communication system to improve capacity and efficiency. By using multiple access techniques, the channels assigned to a cell are shared by mobile stations located in the cell. There are two approaches for channel allocation, viz.

1. Centralized approach in which requests for channel allocation are handled by a central controller that has access to system wide channel usage information $[1,2$, $3,4,8]$.

2. Distributed approach where the base stations and/or the mobile hosts monitor the signal to noise ratio of relevant channels and exchange this information $[5,6,7]$.

Yet, the major problems of wireless communication are limited available bandwidth and more frequent spurious disconnections. Consequently, communication latency rises because of frequent retransmissions, larger time-out delays and more error-control protocol processing [11]. Based on cochannel separation the channel allocation schemes are classified as fixed, dynamic and hybrid.

Fixed Channel algorithm conducts a fixed assignment of channels per base station while for the dynamic channel algorithm (DCA), a base station can handle all the channels for the system and dynamically allocates suitable channels to minimize interference conditions.

In this paper, using dynamic channel allocation scheme, we have examined the carrier to noise ratio concerned with the system and by conducting channel search based on finding an associated low carrier to noise ratio, we show that our system provides a low blocking probability. We have examined the corresponding force termination probability as well.

We begin the next section with describing the system model and the various parameters used.

\section{SYSTEM MODEL}

\section{Allocating available resources}

The system modeling begins with dividing the entire considered geographical area into hexagon cells as shown in fig. 1.

We divided the entire area into 19 cells. Thus we have defined model cluster size of 19 and total channels to be 190 so that each cell contains 10 channels. 


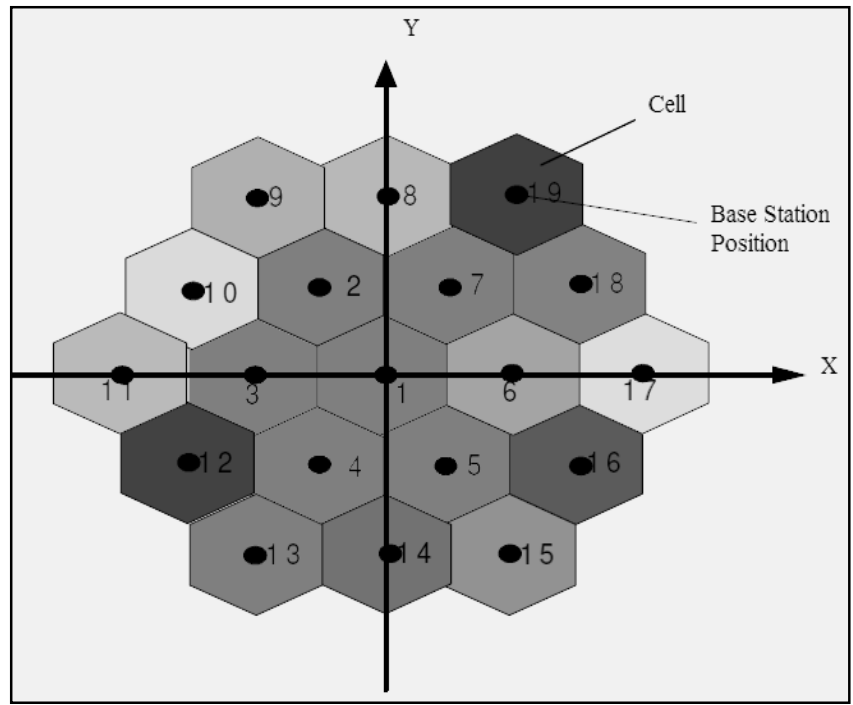

Figure 1: Cell layout showing total 19 cells (with cluster of 7).

\section{Construction of cell layout}

First we have stored the positions of base-stations in a $19 \times 2$ matrix, the two columns storing the $\mathrm{x}$ and $\mathrm{y}$ coordinates of the base station. This is accomplished by taking each cell to be of radius 1 , and calculating all the positions using the fact that each cell is hexagonal.

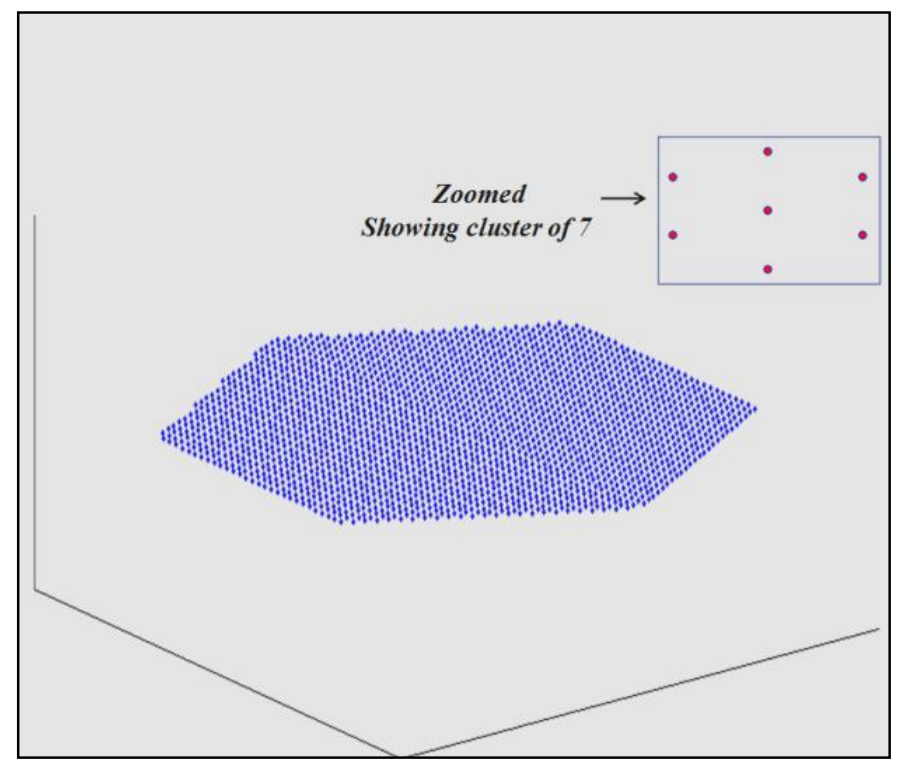

Figure 2: Mesh Pattern for the cell layout

Random location of users has been taken for each of the 19 cells.

Then, Co-channel interference is taken care of using cell wrapping technique. This means that since the environment of boundary cells is different from the intermediate lying cells so the here boundary cells are regarded as the neighbors of boundary cells located almost directly opposite the cell layout.

For Call generation, in each time period we analyze for counting the "not connected" users, using a random function. If this function returns a value greater than our chosen fixed average arrival rate during every time period, then we allow our user to start the call and using a counter we count all such initiated calls.

Also, every initiated call has its own call holding time, and the initiated call is terminated after such a time. The holding time of each call is subject to exponential distribution with a mean value.

\section{Channel assignment}

We begin with analyzing uplink power strength from a user who has initiated call to the base station. Mathematically, this can be obtained by calculating the corresponding carrier to noise ratio. In order to obtain this ratio we have incorporated path loss as well as attenuation due to shadowing.

If the incoming carrier strength in microwatts is $P_{c}$ and the noise level, also in microwatts, is $P_{n}$ then the carrier-to-noise ratio $C / N$ in decibels is given by the formula

$$
\frac{C}{N}=10 \log _{10} \frac{P_{c}}{P_{n}} . \quad . \quad . E q .(1)
$$

And the Carrier to Interference ratio can be obtained as

$$
\frac{C}{I}=\frac{P_{r}}{\sum_{j=1}^{k} P_{\text {of the } j^{\text {th }} \text { interferer }}} \cdot . \text {.Eq.(2) }
$$

By incorporating of contribution from every user that can cause interference and finally adding them up, we obtain the total interference in the denominator of Eq.(2). Now the total carrier to Noise plus Interference ratio can be obtained as follows:

$$
\frac{C}{N+I}=\frac{1}{N / C+I / C}
$$

$$
=\frac{1}{(C / N)^{-1}+(C / I)^{-1}} . . \quad \text { Eq.(3) }
$$

where $(C / N)$ and $(C / I)$ is substituted from Eq.(1) and $E q$.(2) respectively. By analyzing the achieved value of this ratio compared to a threshold value, a call is accepted or blocked.

We have set up a counter for these blocked calls and the results so obtained by varying the number of users per cell are discussed in the next section. 


\section{RESULTS AND DISCUSSION}

The results as calculated for call blocking probability varying as a function of time period is shown in Fig.3

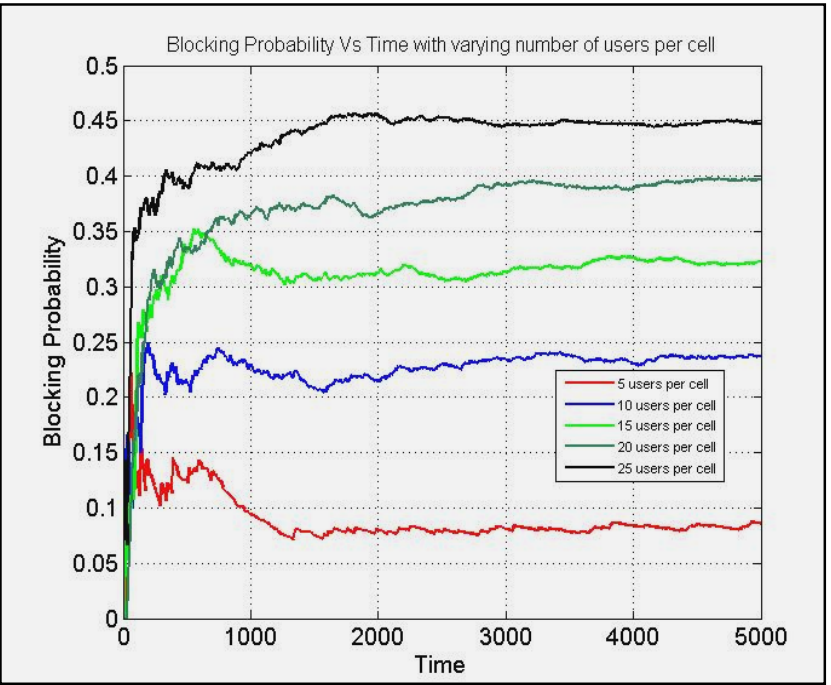

Figure 3: Call blocking probability as a function of time for various number of users per cell

We have compiled our analysis with varying number of users per cell (for 5, 10, 15, 20, 25 users per cell in Fig.3) and the plots shows that after initial fluctuations, our system achieves stable and low blocking probability.

In the next section we bench mark our results with a theoretical analysis.

\section{Theoretical Analysis}

In this section we benchmark the results from the system model with the theoretical model proposed using Engset's loss formula.

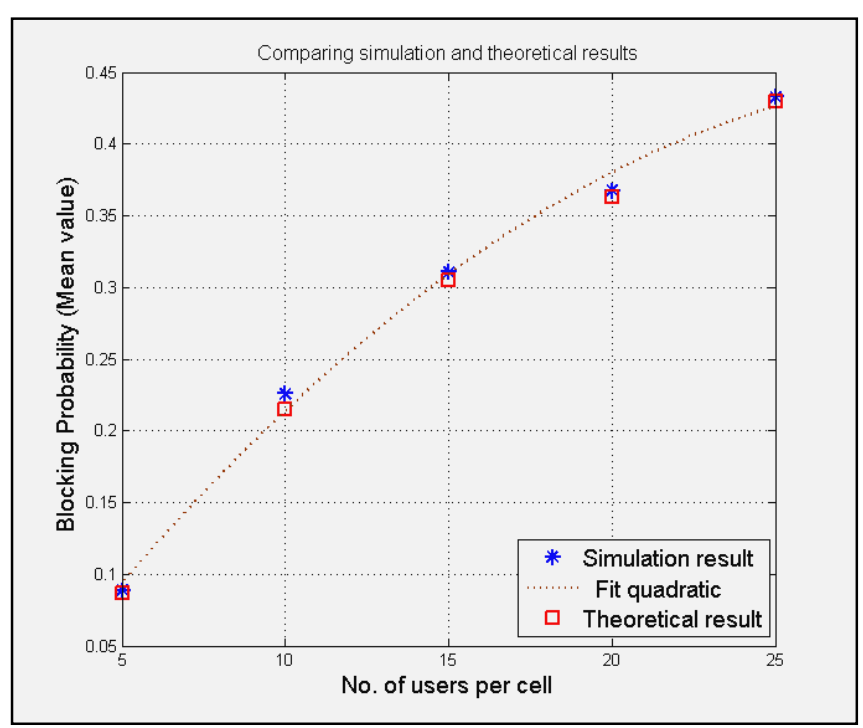

Figure 4: Comparing theoretical and simulation results
Let we Consider $n_{s}$ be the number of users and $n_{c}$ be the number of channels and let $\lambda$ be the mean call arrival rate per user (which is not connected) and $\mu$ be the mean call holding time. Then According to Engset's loss formula

$$
P_{\text {Blocking }}=\frac{\left(\begin{array}{c}
n_{s}-1 \\
n_{c}
\end{array}\right)(\lambda \mu)^{n_{c}}}{\sum_{i=0}^{n_{c}}\left(\begin{array}{c}
n_{s}-1 \\
i
\end{array}\right)(\lambda \mu)^{i}} \cdot . E q .(4)
$$

The mean call blocking probability as calculated from Eq. 4 is plotted in Fig. 4.

The quadratic fit parameter corresponds to the equation $y=p_{1} x^{2}+p_{2} x+p_{3}$ with coefficients

$$
p_{1}=-0.0004908, p_{2}=0.0313324, p_{3}=0.049732
$$

As can be seen from Fig. 4, the theoretical results also agree very well with the results of our system model.

\section{REFERENCES}

[1] M.P. Mishra, P. C. Saxena, "Survey of Channel Allocation Algorithms Research for Cellular System", International Journal of Networks and Communications 2012, 2(5): 75-104

[2] Goutam K. Audhya, Koushik Sinha, Sasthi C. Ghosh and Bhabani P. Sinha., "A survey on the channel assignment problem in wireless networks", WIRELESS COMMUNICATIONS AND MOBILE COMPUTING Wirel. Commun. Mob. Comput. 2011; 11:583-609

[3] Samya Bhattacharya, Hari Mohan Gupta, Subrat Kar, “ Traffic model and performance analysis of cellular mobile systems for general distributed handoff traffic and dynamic channel allocation", IEEE Transactions on Vehicular Technology, Volume 57, Issue 6,3629-3640, 2008.

[4] M. P. Mishra; P. C. Saxena, "Issues, challenges and problems in channel allocation in cellular system", 2011 2nd International Conference on Computer and Communication Technology (ICCCT-2011)

[5] Suyash Kumar, P.V.Suresh "Channel Allocation utilizing Evolutionary Approach", $4^{\text {th }}$ International conference for sustainable global development INDIACOM -2017 at BVICAM, Delhi.

[6] Hale WK. "Frequency assignment: theory and application." Proceedings of the IEEE 1980; 68: 14971514.

[7] Khanbary LMO, Vidyarthi DP. "Reliability based channel allocation using genetic algorithm in mobile 
International Journal of Applied Engineering Research ISSN 0973-4562 Volume 13, Number 5 (2018) pp. 2955-2958 (C) Research India Publications. https://dx.doi.org/10.37622/IJAER/13.5.2018.2955-2958

computing." IEEE Transactions on Vehicular Technology 2009; 58(8):4248-4256.

[8] Suyash Kumar, P.V. Suresh,"Generalized Reduction Approach from 3-SAT to K-Colorability" in International Journal of Emerging Technology \& Advanced Engineering (ISSN 2250-2459,ISO 9001:2008 Certified Journal), Volume 7, Issue 10, October, 2017. 